\title{
Withholding Emotional Expression can Hurt Heart: A Cross-Sectional Study of Attention and Emotion Regulation after Coronary Angioplasty
}

\author{
Anchal Agarwal \\ Ph.D. Research Scholar, Department of Psychology, School of Psychology, Philosophy \& \\ Education, Gujarat University, Ahmedabad, Gujarat, India. \\ Kamayani Mathur \\ Professor \& Head, Department of Psychology, Director, School of Psychology, Philosophy \& \\ Education, Gujarat University, Ahmedabad, Gujarat, India.
}

\begin{abstract}
Emotions and physical health are entwined with each other. Much importance is being given lately in understanding the role of emotions and its management in chronic illnesses, especially cardiovascular disease (CVD), which is one of the leading causes of fatality in the present times. There is already burgeoning literature available with regards to coronary artery bypass grafting(CABG), but scarcely on percutaneous coronary intervention (PCI) patients.
\end{abstract}

Objective: The current study aimed at elucidating the emotion regulation strategy among cardiac patients and their cognitive ability one month after coronary angioplasty as compared to a healthy control group.

Method: A sample of 84 people was selected by using the purposive sampling technique, wherein 42 patients visiting at one-month follow-up after coronary angioplasty treatment belonged to group one and 42 healthy controls in the other. Emotion Regulation Questionnaire (ERQ) by Gross and John (2003) and Comprehensive Trail-Making Test (CTMT) by Cecil R. Reynolds (2002) were used as research tools to collect the data. Administration, scoring and interpretation were done as per the test manuals. MannWhitney U-test was used to statistically analyse the data.

Results and conclusion: The obtained findings indicated that cardiac patients use Expression Suppression $(\mathrm{p}=.001)$ whereas healthy people use Cognitive Reappraisal $(p=.044)$ to regulate their emotions. Furthermore, the angioplasty group performed better than the healthy control group $(\mathrm{p}=.038)$ at the cognitive ability test. Finally, the result also alludes that controlled attention is required for the non-expression of feelings. Thus, the conclusion highlights the need for teaching healthy ER strategies to cardiac patients to alter the detrimental pattern.

Keywords: attention, concentration, coronary angioplasty, emotion regulation, reappraisal, suppression

\section{INTRODUCTION}

According to the modal model, emotions include human-situation transactions that require attention, have significance to the person in the light of currently active priorities, and give rise to organized yet versatile multi-system responses that allow critical adjustments to the ongoing human-situation transaction (J. Gross, 2014). Emotions, therefore, call for a 
systematic collection of behavioral, experiential and physiological response patterns that together affect how we respond to perceived threats and capabilities. Our emotional reactions also coincide immaculately with the challenges of our various situations in life. However, these reactions may also deceive us, particularly when current social and physical environments vary drastically from those that have influenced our emotions over the years. At such times, our emotional reactions can do much more damage than good. When our emotions seem to be out of tune with a given circumstance, we always try to change our emotional reactions to better suit our objectives (J. J. Gross, 2002).

According to Gross (2002), Emotion Regulation (ER) refers to the mechanisms by which we alter the emotions we have and how we feel and communicate them while we have them. As emotions are multi-componential processes that emerge over time, emotional regulation involves changes in "emotional dynamics" or latency, time increase, magnitude, duration, and response offset in behavioral, experiential, or physiological dimensions. Emotion regulation often includes changes in the interrelationship of response elements as the emotion unravels, such as when physiological response increases appear in the absence of observable actions.

An individual can manage his emotion in many varied ways. Cognitive Reappraisal and Expression Suppression are the two most widely used techniques. Both include the downregulation of feeling, which is a standard and appreciated regulatory aim of emotion. Cognitive revaluation is characterized as the reconstruction of a presumably emotional circumstance in non-emotional terms. Expressive Suppression is characterized as preventing the action of ongoing emotional expression. While reappraisal is commonly regarded as an efficient ER method, suppression has proven to be detrimental. In a given setting, suppression reduces whatever behavioral reaction patterns occur with a specific emotion, and heart rate normally accompanies body activity. Electrodermal response and blood pressure are both increased by suppression, whereas finger pulse amplitude, finger temperature, and pulse transit times are decreased. These latter changes are technically consistent and can be defined as "intensified sympathetic activity of the cardiovascular system" (J. J. Gross, 2002).

Regulation of emotions can also influence the risk of cardiovascular disease through direct biological changes associated with the activation of the hypothalamic-pituitary-adrenal axis and sympathetic nervous system. Scientific studies have shown that suppression results in higher activation of the hypothalamic-pituitary-adrenal axis as well as the sympathetic nervous system. Such elevated hypothalamic-pituitary-adrenal axis and sympathetic activation of the nervous system are associated with pathophysiology linked with cardiovascular disease, including endothelium disruption and subsequent inflammation, as well as atherosclerosis and atherosclerotic lipid profiles. Therefore, emotional dysregulation and associated psychosocial stress can facilitate greater hypothalamic-pituitary-adrenal axis, activation of the sympathetic nervous system, and cardiovascular reactivity, while reevaluation and successful emotion and stress regulation can prevent or reduce such behavior. Usage of such proactive versus less efficient regulatory techniques over time will cumulatively modify risk patterns for cardiovascular disease(Appleton, Loucks, Buka, \& Kubzansky, 2014).

For cognitive health, cardiovascular(CV) health has been shown to be significant, e.g. midlife vascular risk variables have been correlated with later mild cognitive deficits as well as dementia. In the life of a person, executive cognitive functioning (including processes of working memory, attentional and cognitive control processes such as inhibition, shifting attention, updating and maintenance) is important. At the same time, executive cognitive functioning differs over time between people as well as within persons and tends to be especially susceptible to deficits in physiological processes caused by stress and wear and tear with aging. In approaches for facilitating and sustaining executive cognitive functioning throughout life, knowledge of the processes that influence executive cognitive functioning is therefore relevant and crucial. 
It is probable that autonomic regulation and CV physiology are the fundamental factors influencing the cognitive abilities of individuals, for example as a consequence of psychosocial/psychological stress. Notably, autonomic regulation can be affected with longterm stress exposure, which in turn has detrimental effects on CV health as well as cognitive health. Related effects also involve decreased stress resilience, i.e. decreased efficient and versatile response to problems, enhanced or altered emotional and physiological responses to stressors, which may further lead to CV and brain health degradation. The findings from previous literature seem to demonstrate a trend of better CV regulation that is primarily linked with improved cognitive inhibition, shifting, updating, and speed in some cases. Cognitive functions are thus closely related to cardiac health (Stenfors, Hanson, Theorell, \& Osika, 2016).

Attention is the most essential tool that acts as a telescope from which the stimuli we encounter in our environment are picked, brought into focus, and magnified. Via numerous attentional mechanisms, relevant sensory, emotional, and mental information is filtered, construed and evaluated, which can be regulated automatically or deliberately. Evidently, what we attend to, either good or bad, will influence our perceptions. How effective people are in manipulating their attentional processes may dictate their consequent effective experience and behavioral pathways. While there are individual variations in the ability to regulate attention, recent research has indicated that through consistent practice, the mechanisms involved in attention modulation can be learned and strengthened. If attention can be conditioned, then it can be used to effectively direct the strategies of emotion regulation and downstream behavior of individuals, thereby improving subjective wellbeing - in other words, in order to optimize their emotional experience, individuals could practice to selectively focus on specific types of information (Wadlinger \& Isaacowitz, 2011).

\section{Objectives}

The main aim of this research is to study and compare the emotion regulation strategies primarily used by cardiac patients who have undergone the treatment of coronary angioplasty with the healthy control group who have no history of any cardiac issues. The study also aims at evaluating the attention/concentration ability of both the groups and further observe if it contributes in some way to the emotional regulation strategy dominantly practiced by them.

\section{Null Hypotheses}

1. There is no significant difference between the angioplasty group and control group for using cognitive reappraisal as a strategy to regulate their emotions.

2. There is no significant difference between the angioplasty group and control group for using expression suppression as an emotion regulation strategy.

3. The angioplasty group and control group do not differ significantly on the attention/concentration domain of their cognitive ability.

\section{METHODOLOGY \\ Selection of the sample}

The total sample consisted of 84 persons $(42$ people in each respective group, i.e., the coronary angioplasty group and the control group) and were in the age range of $25-74$ years comprising of both genders. A purposive sampling technique was used to collect the sample from a hospital of Ahmedabad city. Here in this research study, the participants belonging to the angioplasty group were approached when they would come for their onemonth follow-up after the treatment and those in the healthy control group were selected keeping in mind that they have no history of CVH issues and those who were not diagnosed with any of the chronic illnesses, i.e., hypertension, hypercholesterolemia and diabetes mellitus.

\section{Variables}

\section{Independent variable}

1. Health status of the participant, i.e., Angioplasty group and healthy control group 


\section{Dependent variables}

1. Emotion Regulation (Cognitive reappraisal and Expression suppression)

2. Attention andconcentration

\section{Research measures}

- Inclusion criteria

1. People in the age range of $25-74$ years were only selected as a sample for the present study.

2. People of both genders were included to be a part of the study.

3. An equal number of angioplasty patients, as well as healthy people, were screened out and selected for respective groups.

4. The participants in the angioplasty group were selected only at the time when they came for their one-month follow-up after the treatment.

- Exclusion criteria

1. Persons not knowing basic English to understand and perform the tests were excluded from the study.

2. People with any sort of physical illness, chronic disease, or cardiovascular health issue were excluded from being part of the healthy control group.

3. The trail-making test was not continued with those participants who could not understand and complete tracing the sample trail.

4. People with any problem related to motor functioning of their dominant hand or issues related to vision were excluded from taking the performance test.

\section{Research tools}

In the present research study, the following tools were used for the purpose of data collection:

\section{Emotion Regulation Questionnaire}

\section{Author: Gross, J.J., \& John, O.P. (2003)}

The Emotions Regulation Questionnaire (ERQ) items were rationally derived and indicated clearly in each item is the emotion regulatory process intended for measurement. In addition to these general-emotion items, the Reappraisal scale and the Suppression scale both included at least one item asking about regulating negative emotion (illustrated for the participants by giving sadness and anger as examples) and one item about regulating positive emotion (exemplified by joy and amusement). Moreover, care was taken to limit the item content to the intended emotion regulatory strategy, and to avoid any potential confounding by mentioning any positive or negative consequences for affect, social functioning, or well-being. The final 10 items are rated on a scale from 1 (strongly disagree) to 7 (strongly agree). Alpha reliabilities averaged .79 for Reappraisal and .73 for Suppression. Test-retest reliability across 3 months was .69 for both scales. It does not take more than 10 minutes to complete the entire questionnaire.

\section{Comprehensive Trail-Making Test (C-TMT)}

Author: Cecil R. Reynolds (2002)

The C-TMT is a standardized set of five visual search and sequencing tasks. Attention, concentration, resistance to distraction, and cognitive flexibility (or set-shifting) heavily influence performance on these tasks. The respondent connects a series of stimuli in a specified order as quickly as possible(Reynolds, 2002).

Age group: The test can be used for people belonging to the age range of 8 through 74 years and 11 months.

Administration: According to the test manual, the time required to administer the entire CTMT ranges from approximately 5 to 12 minutes. The five trails of the CTMT are to be administered in numerical order only.

Scoring and Interpretation: The raw score derived for each trial is the number of seconds used to complete each trial. To derive the $T$-score for each trail, transfer the time in seconds 
for each trial using the manual. A composite score is then obtained by pooling the $T$ scores from the individual trails. Qualitative description of performance levels expressed as $T$ scores on the CTMT trails and the CTMT composite index is described in the test manual.

Reliability: The test-retest reliability is .84 , the internal consistency coefficient is .91 and the scorer reliability is .99 for this performance test.

Validity: The CTMT tasks receive strong theoretical support from the models of brain systems and also from decades of research on the original trail-making tasks. Comparisons of the CTMT tasks with the constructs of attention, executive control, visual search, and sequencing show a high degree of concordance.

\section{Data collection and procedure}

To obtain the sample group, official permission was taken from the authorities in charge of a hospital in Ahmedabad city, Gujarat. Followed by a brief rapport, informed consent was obtained from each subject promising confidentiality and anonymity. The demographic data sheet was first given to the participants to obtain the required information. Research tools were then administered after giving relevant instructions and ensuring that the subject has understood them. The emotional regulation questionnaire was given first followed by the trail-making test. Only trails 1 to 3 were used to assess the attention and concentration variable. Privacy and a comfortable atmosphere were ensured throughout the data collection. All the subjects were thanked for giving their valuable time and consent to participate in the study. After the completion of the data collection process, which took approximately six months to obtain this sample, the performance of each respondent on the test was scored using the test manual.

\section{Statistical analysis}

Descriptive statistics were computed and then Mann-Whitney U-test was conducted to statistically analyse the data for both the groups on the given measures. Further, effect size (r) was calculated for each measure using Cohen's formula for the Mann-Whitney test (Cohen, 2013)to find out the practical significance of the obtained results.

\section{RESULTS}

The findings of this research study have been interpreted and presented in form of tables and graphs below:

Table no. 1: Shows the descriptive statistics for both groups on the given measures.

\begin{tabular}{lllll}
\hline \multirow{3}{*}{ Participant group } & & $\begin{array}{l}\text { Cognitive } \\
\text { Reappraisal }\end{array}$ & $\begin{array}{l}\text { Expression } \\
\text { Suppression }\end{array}$ & $\begin{array}{l}\text { Attention/ } \\
\text { Concentratio } \\
\text { Angioplasty group }\end{array}$ \\
\cline { 2 - 5 } & $\mathrm{N}$ & 42 & 42 & 42 \\
\cline { 2 - 5 } & Mean & 28.64 & 18.55 & 33.03175 \\
\cline { 2 - 5 } & Median & 30.00 & 20.00 & 33.50000 \\
\cline { 2 - 5 } & Std. Deviation & 6.771 & 5.718 & 6.379511 \\
\cline { 2 - 5 } & Variance & 45.845 & 32.693 & 40.698 \\
\cline { 2 - 5 } & Skewness & -.564 & -.624 & -.301 \\
\cline { 2 - 5 } Control group & Kurtosis & -.519 & -.726 & -.494 \\
\cline { 2 - 5 } & $\mathrm{N}$ & 42 & 42 & 42 \\
\cline { 2 - 5 } & Mean & 32.12 & 14.21 & 30.30159 \\
\cline { 2 - 5 } & Median & 32.00 & 15.00 & 30.66667 \\
\cline { 2 - 5 } & Std. Deviation & 6.371 & 5.585 & 5.888673 \\
\cline { 2 - 5 } & Variance & 40.595 & 31.197 & -.0424 \\
\cline { 2 - 5 } & Skewness & -.080 & -.776 & -.163 \\
\cline { 2 - 5 } & Kurtosis & -.993 & & -.044 \\
\hline \hline
\end{tabular}


As shown in table no.1, the data was skewed (not normally distributed) so the most appropriate statistical test was Mann-Whitney U to statistically analyse the data.

Table no.2: Shows the result of the Mann-Whitney U-Test and its effect size for the
given measures. (a) Ranks

\begin{tabular}{lllll}
\hline \hline & & & Sum & of \\
& Participant group & $\mathrm{N}$ & Mean Rank & Ranks \\
\hline Cognitive & Angioplasty group & 42 & 37.15 & 1560.50 \\
Reappraisal & Control group & 42 & 47.85 & 2009.50 \\
& Total & 84 & & \\
\hline Expression & Angioplasty group & 42 & 51.74 & 2173.00 \\
Suppression & Control group & 42 & 33.26 & 1397.00 \\
& Total & 84 & & \\
\hline Attention/ & Angioplasty group & 42 & 48.01 & 2016.50 \\
Concentration & Control group & 42 & 36.99 & 1553.50 \\
& Total & 84 & & \\
\hline \hline
\end{tabular}

(b) Test Statistics

\begin{tabular}{|c|c|c|c|}
\hline & $\begin{array}{l}\text { Cognitive } \\
\text { Reappraisa } \\
1\end{array}$ & $\begin{array}{l}\text { Expression } \\
\text { Suppression }\end{array}$ & $\begin{array}{l}\text { Attention/ } \\
\text { Concentratio } \\
\mathrm{n}\end{array}$ \\
\hline Mann-Whitney U & 657.500 & 494.000 & 650.500 \\
\hline Wilcoxon $\mathrm{W}$ & 1560.500 & 1397.000 & 1553.500 \\
\hline$Z$ & -2.012 & -3.477 & -2.072 \\
\hline Asymp. Sig. (2-tailed) & .044 & .001 & .038 \\
\hline Effect size $r$ & -0.21953 & -0.37937 & -0.22607 \\
\hline$r^{2}$ & 0.048192 & 0.143923 & 0.051109 \\
\hline
\end{tabular}

As the result in table no.2 shows, a significant difference was found for the cognitive reappraisal domain of emotional regulation strategy with mean ranks for Angioplasty group and Control group being 37.15 and 47.85 respectively; $U=657.500, Z=-2.012, p=.044, r$ $=-0.219$. This shows that there is a statistically significant difference at 0.05 level $(p<.05)$ between both the groups with respect to cognitive reappraisal as an emotional regulation strategy, wherein the angioplasty group has a lesser mean rank than the control group. Also, the effect size for the same shows that the difference between both groups is $4.8 \%$ which is small according to Cohen's classification of effect size. Thus, the result clearly states that the healthy control group is better at regulating their emotions using cognitive reappraisal strategy as compared to the cardiac patients who have gone under angioplasty treatment, which rejects the null hypothesis.

Further, the descriptive statistics, as well as table no.2, shows that the angioplasty group (median=20; mean rank=51.74) scored higher on expression suppression than the control group (median=15; mean rank=33.26). Mann-Whitney U-value was found to be statistically significant $U=494(Z=-3.477), p<0.01$, and the difference between the two groups is medium $(\mathrm{r}=-0.379)$ with an effect size of $14 \%$. Thus, the obtained result rejects the null hypothesis indicating that the angioplasty group uses expression suppression strategy more than the control group to regulate their emotions.

For the attention/concentration measure, the result tables evidently demonstrate that the angioplasty group (median=33.5; mean rank=48.01) scored more than the control group (median=30.66; mean rank=36.99). Mann-Whitney U-value was found to be statistically significant at 0.05 level with the value of $U=650.5(Z=-2.072), p=.038$, and the difference between the two groups is small $(r=-0.226)$ with an effect size of $5.1 \%$. Thus, it can be inferred from the obtained values that the null hypothesis is rejected as the group of 
patients who had undergone angioplasty treatment when tested on the attention/concentration measure at one-month follow-up, performed better than the healthy control group showing the better cognitive ability for this specific domain.

\section{Figure no.1: Shows the graphical representation of the mean values for both groups on all the given measures.}

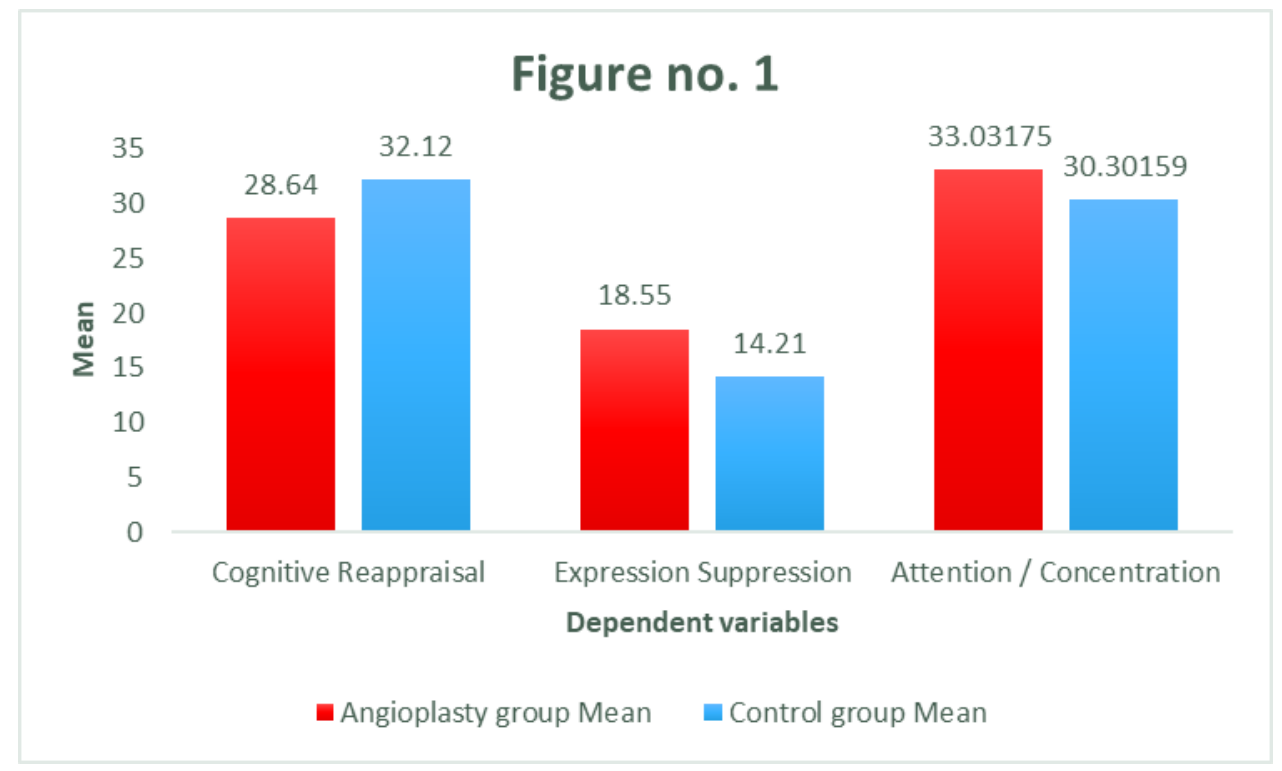

Figure no. 1 shows the mean values of the angioplasty group and control group for the emotional regulation domains and the attention/concentration measure. The values presented in the graph clearly depicts that the cardiac patients' group who have undergone angioplasty have proved to choose more to suppress the expression of their emotions as a strategy to handle their feelings whereas the healthy control group has appeared to use cognitively reappraising their emotions more when dealing with them. On the other hand, the angioplasty group has performed better on the attention/concentration measure in comparison to the control group, when tested at their one-month follow-up after the treatment.

Figure no.2: Shows the comparison of the trend line for both groups on the given measures.

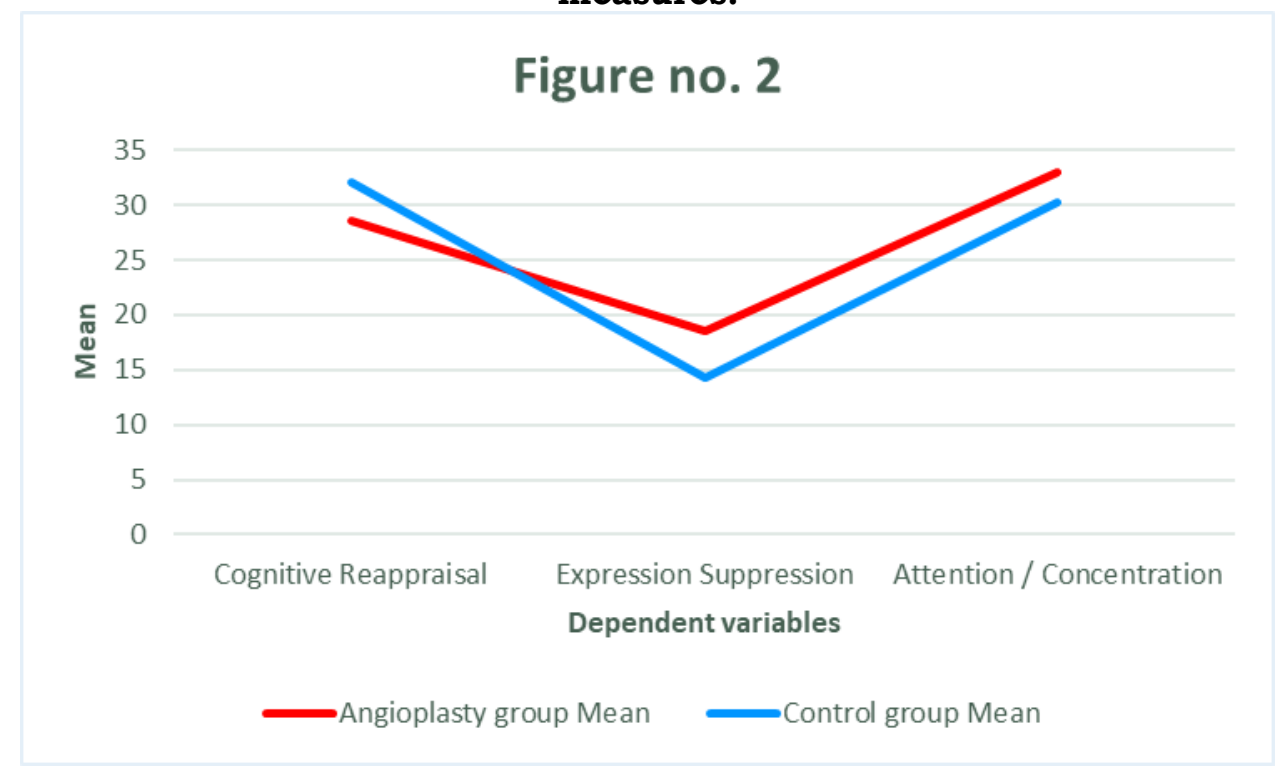


Figure no. 2 illustrates the pattern for the angioplasty group and control group for the strategies they use. The angioplasty group is higher on expression suppression strategy and low on cognitive reappraisal strategy to regulate emotions as contrasted to the healthy control group suggesting their primary approach while dealing with their emotions. However, the angioplasty group has executed superior cognitive ability in the domain of attention/concentration as compared to the control group which contradicts the finding of the use of emotion regulation strategy used by them, as proved by many comparable investigations in the same area. This obtained result from the current study will thus be discussed and supported in the light of previous studies in the discussion below.

\section{Discussion}

The concept that poorly managed negative feelings can cause illnesses has long been identified by health psychologists. There are a number of research findings supporting this argument. Hypertension and coronary heart disease are associated with both chronic hostility and restricting anger. The basic idea is that strong control of negative feelings may have a negative impact on physical health (J. J. Gross, 1998). In a study conducted by Roy, Riley, and Sinha (2018), it was found that chronic stress among those with impaired emotion regulation was correlated with $\mathrm{CV}$ risk factors, indicating that enhancing approaches for emotion regulation earlier in life, during childhood, adolescence, and young adulthood may have long-term benefits in preventing mortality and morbidity from the elevated risk of CV illness (Roy, Riley, \& Sinha, 2018).

The results of the current study show that cardiac patients who have undergone coronary angioplasty procedures use suppression of the expression of their emotional responses as a primary technique to control their feelings, while cognitive reappraisal was seen by the healthier control group as a predominantly used strategy to typically manage their emotions. There are several parallel studies supporting this outcome and giving the same rationale. For instance, in cardiac patients and the general population, Mirlohi and colleagues (2017) studied emotion control strategies (reappraisal and suppression). Results showed that heart disease patients prefer suppression, and reappraisal was commonly used by the control group to modify their emotions (Mirlohi, Mohajerin, Mirlohi, \& Aref, 2017). Another such research by Bahremandand colleagues (2016) reinforces the view that people with heart disease are highly susceptible to aspects of pathology, using contradictory methods to cope with life issues and adverse conditions, so it can be said that these techniques are unfavourable risk factors for heart disease (Bahremand, Alikhani, Zakiei, Janjani, \& Aghei, 2016). It can be seen that having ineffective emotion regulation techniques is a risk factor for falling prey to cardiovascular problems, according to the findings of their research; hence, it is recommended to assist the wellbeing of the population by identifying and rectifying emotion regulation strategies in people in this realm. In yet another investigation conducted by Appleton and colleagues (2014), reevaluation was reported to be linked with a lower 10-year risk of cardiovascular disease, while suppression was related to a higher 10-year risk of cardiovascular disease. These findings were comparable with earlier work in this group and other population-based research to find cardiovascular health-related emotion regulation (Appleton, Loucks, Buka, \& Kubzansky, 2014).

In addition, a plethora of studies were carried out on CV risk factors as well as CHD and its association with cognitive and executive skills, but most of them were done for such studies with CABG patients as the target group. There are too few studies available that have taken coronary angioplasty patients into account while conducting such studies alone. In order to bridge the gap in available information, the present study focused explicitly on this overlooked population. In such studies, more emphasis has also been put on the medical or physiological aspect, e.g. the treatment process itself and how it affects the long-term cognitive functioning of patients (Sauër et al., 2013; Berger et al., 2015), but hardly any studies have concentrated on the psychological dimensions when researching cognition among patients with CVD. 
In researching the influence of CVD on cognition, numerous research studies have found conflicting findings. Some researchers contend that low cognitive output is linked with cardiovascular risk factors (Rumy, Takeda, Martins Matos, \& Nery De Souza-Talarico, 2017), although many others have found contradictory evidence and proved that cardiac functions are not connected to executive functions (Eggermont et al., 2017). Different investigators have also presented data that CAD patients have poorer cognitive baseline output and greater deterioration over the initial period relative to their healthy counterparts, but when reassessed over a long period of time, they do not vary substantially from them on their cognitive test scores (McKhann et al., 2005; Selnes et al., 2009).In the first year following CABG surgery, a meta-analysis of cognitive outcomes following coronary artery bypass graft surgery by Cormack and co-researchers (2012) indicated enhancement in cognitive function. This goes against the more negative view of the findings of many other studies, which may indicate poor outcomes in a few patients and/or methodological problems (Cormack et al., 2012).

Finding of the current research shows that when tested at their one-month follow-up after the non-invasive procedure, the angioplasty group performed better at the trail-making test compared to the heart-healthy control group, indicating that they had better post-PCI attention and concentration abilities even after only a month of the treatment period. This research is in line with another Agarwal, A. and Mathur, K. (2020) study that concluded that cardiac patients (identified on the basis of their angiography reports and prescribed medications) and healthy people differ in their cognitive functioning, where the former group showed better cognitive output compared to the latter group(Agarwal \& Mathur, 2020). There are also several other reports on CABG patients that have shown similar significant post-surgery improvement in patients' cognitive levels, even in a short period of time, benefiting their cognitive function as well as their overall health (Bruce, Yelland, Smith, \& Robinson, 2013; Hogan, Shipolini, Brown, Hurley, \& Cormack, 2013).

As previously noted, that emotion and physical health are closely linked, the emotional well-being of a person depends on the ability to effectively participate in a variety of coping mechanisms to manage affective responses. In view of its prominence among cardiac patients as discussed above and its connection with executive functions (Wadlinger \& Isaacowitz, 2011), the current study further observes that the angioplasty group whose prime ER technique is expression suppression happens to have better attention and concentration capacity compared to the control group that mainly uses reassessment to manage their emotions. The annotation is conflicting, as in a recent report, which argues that self-directed focus helps to reduce negative emotions as well as to reduce emotional scores, thereby increasing emotional well-being among those who normally use expression suppression (Dolcos et al., 2020).

A Webb and colleagues (2012) meta-analysis examined the efficacy of emotion regulation process model techniques in altering emotional consequences as documented by perceptual, behavioral, and biological measures. The results of their research showed that distraction was an efficient way to regulate emotions, while the focus was not. On the basis of this examination, it can be deduced that the finding obtained in the present study indicates that distraction can be more effective as a method of attentional deployment because it enables individuals to disengage from the strong negative stimulus or when they have no other alternative to choose from (Webb, Miles, \& Sheeran, 2012). Whereas focus or inhibition of distraction can potentially lead to a rumination of negative emotions, which is ultimately a deleterious tendency to cope with unpleasant feelings. It is also reasonable to state that focused attention is needed to prevent an individual's expression of feelings and to use restriction of emotional communication as a form of regulatory tool.

Emotion suppression (or non-expression) has lately attracted much interest in the study of the relationship between emotions and health, particularly in the area of chronic disease, as an ER tool. This relates to the alteration of the behavioral component of the emotional experience (i.e. inhibition) (Karademas, Tsalikou, \& Tallarou, 2011). In this domain, an interesting point of incongruity has arisen: a lasting impact of negative emotions is 
observed for suppression but not reappraisal (Ortner, Zelazo, \& Anderson, 2013) and while suppressing negative emotions left the negative emotion experience consistent, suppressing positive emotions reduced the intensity of these emotions (J. J. Gross \& John, 2003). The intense physiological effects of suppression have been the subject of several experimental investigations to date. However, since there tend to be consistent individual variations in methods of emotion regulation, such distinctions are mostly predicted to have aggregate repercussions. For instance, physiological responses are amplified each time emotions are suppressed. It is unlikely that any single physiological reaction of elevated severity would have detrimental impacts. But if such reactions reappear day after day after day, harmful health effects can result (J. J. Gross, 2002).

To sum up, numerous studies have shown that the use of reappraisal to manage emotions is related with healthier patterns of effect, social functioning, and well-being than the use of suppression. During adult years, a deliberative change towards a progressively healthy emotional regulation pattern (i.e. more use of reappraisal and less use of suppression) can be beneficial in coping with any chronic diseases, especially CVD, at the psychological level (John \& Gross, 2004).

\section{Conclusion}

The current study indicates that cardiac patients choose to suppress the expression of their emotions over reappraisal as their main regulatory strategy which adds to the existing literature that having an unhealthy pattern of modifying feelings can contribute as a CV risk factor, damaging the heart over the long run and be one of the potential causes of CHD in those individuals. Also, they show better performance as compared to healthy heart controls in their specific cognitive abilities post-PCI treatment which implies that the negative outcome from various studies needs some reconsideration while taking more PCI patients as the study sample. Moreover, this study proposes that inhibiting emotional expression requires more controlled attention than reassessing the feelings.

\section{Limitations}

As this is a cross-sectional study, no causal inferences can be made. The sample size is not very large and also contains participants from the hospital of Ahmedabad city only which does not allow the generalization of the findings to the larger population. Even though gender and age are not considered as independent variables in the present study, there is an over-representation of males and older adults in the sample as compared to female participants and young adults and it might have influenced the result otherwise if these factors were regarded. Moreover, due to time constraints, the measures were given just at one point of time to the patients in the post-treatment condition, without a baseline score.

\section{Suggestions}

The same study can be done on a larger and diverse sample to obtain a more accurate result. Additionally, gender and age factors can be taken into consideration and a uniform representation of the participants on these variables in the total sample can further help to attain even precise findings for a better understanding of the research objective. Besides, follow-ups can be done at different time stamps for better comparison on the trail-making measure and observe if their performance changes over the time period after the treatment. Furthermore, other ER strategies can also be studies apart from the two strategies highlighted in this paper.

\section{Practical Implications}

There are not many studies done of this kind in India taking patients of coronary angioplasty into consideration, more light has always been focused on CABG patients usually. Thus, this study helps to understand the operating mechanism of the angioplasty patients at the emotional level. Also, attention and concentration as one of the executive functions have been studied specifically at one-month follow-up after the angioplasty to find out how they perform on the measure in the post-treatment condition when they are recovering from the coronary artery blockage. The current investigation also highlights the influence of attention on the strategies chosen by the angioplasty group as compared to the 
healthy control group for regulating their emotions on day to day basis. Overall, the findings contribute to the knowledge that health psychologists and cardiologists along with other medical professionals can use to benefit the cardiac patients and design modified interventions to build adaptive emotion regulation skills and reduce less adaptive strategies as well as useful for cardiac rehabilitation programs to help patients achieve a comprehensive recovery from the illness and not just focus on treating the physiological aspect of the ailment.

\section{References}

[1]. Agarwal, A., \& Mathur, K. (2020). An Analysis of Cognitive Functioning Among Cardiac Patients After Coronary Angiography. AEGAEUM Journal, 8(7), 80-93. Retrieved from http://aegaeum.com/

[2]. Appleton, A. A., Loucks, E. B., Buka, S. L., \&Kubzansky, L. D. (2014). Divergent Associations of Antecedent- and Response-Focused Emotion Regulation Strategies with Midlife Cardiovascular Disease Risk. Annals of Behavioral Medicine, 48(2), 246. https://doi.org/10.1007/s12160-014-9600-4

[3]. Bahremand, M., Alikhani, M., Zakiei, A., Janjani, P., \&Aghei, A. (2016). Emotion Risk-Factor in Patients with Cardiac Diseases: The Role of Cognitive Emotion Regulation Strategies, Positive Affect and Negative Affect (A Case-Control Study). Global Journal of Health Science, 8(1), 173179. https://doi.org/10.5539/gjhs.v8n1p173

[4]. Berger, M., Nadler, J. W., Browndyke, J., Terrando, N., Ponnusamy, V., Cohen, H. J., ... Mathew, J. P. (2015, September 1). Postoperative Cognitive Dysfunction. Minding the Gaps in Our Knowledge of a Common Postoperative Complication in the Elderly. Anesthesiology Clinics, Vol. 33, pp. 517-550. https://doi.org/10.1016/j.anclin.2015.05.008

[5]. Bruce, K. M., Yelland, G. W., Smith, J. A., \& Robinson, S. R. (2013). Recovery of cognitive function after coronary artery bypass graft operations. Annals of Thoracic Surgery, 95(4), 1306-1313. https://doi.org/10.1016/j.athoracsur.2012.11.021

[6]. Cohen, J. (2013). Statistical Power Analysis for the Behavioral Sciences. In Statistical Power Analysis for the Behavioral Sciences. https://doi.org/10.4324/9780203771587

[7]. Cormack, F., Shipolini, A., Awad, W. I., Richardson, C., McCormack, D. J., Colleoni, L., ... Hogan, A. M. (2012, October). A meta-analysis of cognitive outcome following coronary artery bypass graft surgery. Neuroscience and Biobehavioral Reviews, Vol. 36, pp. 2118-2129. https://doi.org/10.1016/j.neubiorev.2012.06.002

[8]. Dolcos, F., Bogdan, P. C., O’Brien, M., Iordan, A. D., Madison, A., Buetti, S., ... Dolcos, S. (2020). The impact of focused attention on emotional evaluation: An eye-tracking investigation. Emotion. https://doi.org/10.1037/emo0000895

[9]. Eggermont, L. H. P., Aly, M. F. A., Vuijk, P. J., de Boer, K., Kamp, O., van Rossum, A. C., \&Scherder, E. J. A. (2017). Cardiac function and cognition in older community-dwelling cardiac patients. Psychogeriatrics, 17(6), 356-363. https://doi.org/10.1111/psyg. 12245

[10]. Gross, J. (2014). Emotion Regulation: Conceptual and Empirical Foundations. In Handbook of Emotion Regulation, Second edition (pp. 3-20).

[11]. Gross, J. J. (1998). The emerging field of emotion regulation: An integrative review. Review of General Psychology, 2(3), 271-299. https://doi.org/10.1037/1089-2680.2.3.271

[12]. Gross, J. J. (2002). Emotion regulation: Affective, cognitive, and social consequences. Psychophysiology, Vol. 39, pp. 281-291. https://doi.org/10.1017/S0048577201393198

[13]. Gross, J. J., \& John, O. P. (2003). Individual Differences in Two Emotion Regulation Processes: Implications for Affect, Relationships, and Well-Being. Journal of Personality and Social Psychology, 85(2), 348-362. https://doi.org/10.1037/0022-3514.85.2.348

[14]. Hogan, A. M., Shipolini, A., Brown, M. M., Hurley, R., \& Cormack, F. (2013, July 9). Fixing hearts and protecting minds: A review of the multiple, interacting factors influencing cognitive function after coronary artery bypass graft surgery. Circulation, Vol. 128, pp. 162-171. https://doi.org/10.1161/CIRCULATIONAHA.112.000701

[15]. John, O. P., \& Gross, J. J. (2004). Healthy and unhealthy emotion regulation: Personality processes, individual differences, and life span development. Journal of Personality, Vol. 72, pp. 1301-1334. https://doi.org/10.1111/j.1467-6494.2004.00298.x

[16]. Karademas, E. C., Tsalikou, C., \&Tallarou, M. C. (2011). The impact of emotion regulation and illness-focused coping strategies on the relation of illness-related negative emotions to subjective health. Journal of Health Psychology, 16(3), 510-519. https://doi.org/10.1177/1359105310392093

[17]. McKhann, G. M., Grega, M. A., Borowicz, L. M., Bailey, M. M., Barry, S. J. E., Zeger, S. L., ... Selnes, O. A. (2005). Is there cognitive decline 1 year after CABG? Comparison with surgical and nonsurgical controls. Neurology, 65(7), 991-999. https://doi.org/10.1212/01.wnl.0000175220.78475.99 
[18]. Mirlohi, S. M. R., Mohajerin, B., Mirlohi, M. J., \&Aref, N. M. (2017). Differences Between Expressive Suppression and Cognitive Reappraisal Between Heart Disease and General Population. International Journal of Biomedicine, 7(3), 192-195. https://doi.org/10.21103/article7(3)_oa6

[19]. Ortner, C. N. M., Zelazo, P. D., \& Anderson, A. K. (2013). Effects of emotion regulation on concurrent attentional performance. Motivation and Emotion, 37(2), 346-354. https://doi.org/10.1007/s11031-012-9310-9

[20]. Reynolds, C. R. (2002). Comprehensive trail making test (CTMT): Examiner's manual. Austin, Texas: PRO-ED.

[21]. Roy, B., Riley, C., \& Sinha, R. (2018). Emotion regulation moderates the association between chronic stress and cardiovascular disease risk in humans: a cross-sectional study. Stress, 21(6), 548-555. https://doi.org/10.1080/10253890.2018.1490724

[22]. Rumy, J., Takeda, T., Martins Matos, T., \& Nery De Souza-Talarico, J. (2017). Cardiovascular risk factors and cognitive performance in aging. Dement Neuropsychol, 11(4), 442-448. https://doi.org/10.1590/1980-57642016dn11-040015

[23]. Sauër, A. M. C., Nathoe, H. M., Hendrikse, J., Peelen, L. M., Regieli, J., Veldhuijzen, D. S., ... Van Dijk, D. (2013). Cognitive outcomes 7.5 years after angioplasty compared with off-pump coronary bypass surgery. Annals of Thoracic Surgery, 96(4), 1294-1300. https://doi.org/10.1016/j.athoracsur.2013.05.001

[24]. Selnes, O. A., Grega, M. A., Bailey, M. M., Pham, L. D., Zeger, S. L., Baumgartner, W. A., \&McKhann, G. M. (2009). Do Management Strategies for Coronary Artery Disease Influence 6Year Cognitive Outcomes? Annals of Thoracic Surgery, 88(2). https://doi.org/10.1016/j.athoracsur.2009.04.061

[25]. Stenfors, C. U. D., Hanson, L. M., Theorell, T., \&Osika, W. S. (2016). Executive cognitive functioning and cardiovascular autonomic regulation in a population-based sample of working adults. Frontiers in Psychology, 7(OCT), 1536. https://doi.org/10.3389/fpsyg.2016.01536

[26]. Wadlinger, H. A., \&Isaacowitz, D. M. (2011). Fixing our focus: Training attention to regulate emotion. Personality and Social Psychology Review, 15(1), 75-102. https://doi.org/10.1177/1088868310365565

[27]. Webb, T. L., Miles, E., \& Sheeran, P. (2012). Dealing with feeling: A meta-analysis of the effectiveness of strategies derived from the process model of emotion regulation. Psychological Bulletin, 138(4), 775-808. https://doi.org/10.1037/a0027600 